\title{
Role of histone deacetylase 2 and its posttranslational modifications in cardiac hypertrophy
}

\author{
Gwang Hyeon Eom ${ }^{*} \mathcal{E}$ Hyun Kook ${ }^{*}$ \\ Department of Pharmacology and Medical Research Center for Gene Regulation, Chonnam National University Medical School, Gwangju \\ 501-746, Korea
}

Cardiac hypertrophy is a form of global remodeling, although the initial step seems to be an adaptation to increased hemodynamic demands. The characteristics of cardiac hypertrophy include the functional reactivation of the arrested fetal gene program, where histone deacetylases (HDACs) are closely linked in the development of the process. To date, mammalian HDACs are divided into four classes: I, II, III, and IV. By structural similarities, class II HDACs are then subdivided into lla and IIb. Among class I and II HDACs, HDAC2, 4, 5, and 9 have been reported to be involved in hypertrophic responses; HDAC4, 5, and 9 are negative regulators, whereas HDAC2 is a pro-hypertrophic mediator. The molecular function and regulation of class Ila HDACs depend largely on the phosphorylation-mediated cytosolic redistribution, whereas those of HDAC2 take place primarily in the nucleus. In response to stresses, posttranslational modification (PTM) processes, dynamic modifications after the translation of proteins, are involved in the regulation of the activities of those hypertrophy-related HDACs. In this article, we briefly review 1) the activation of HDAC2 in the development of cardiac hypertrophy and 2) the PTM of HDAC2 and its implications in the regulation of HDAC2 activity. [BMB Reports 2015; 48(3): 131-138]

\section{INTRODUCTION}

Cardiac hypertrophy is an adaptation process to meet the increased hemodynamic demands in peripheral tissues due to underlying diseases, such as hypertension, valve stenosis or regurgitation, or even myocardial infarction (1-3). The characteristics are an increase in cellular burden of individual car-

*Corresponding authors. Hyun Kook, Tel: +82-62-220-4242; Fax: +8262-232-6974; E-mail: kookhyun@chonnam.ac.kr, Gwang Hyeon Eom, Tel: +82-62-220-4237, Fax: +82-62-232-6974, E-mail: eomgh @chonnam.ac.kr

http://dx.doi.org/10.5483/BMBRep.2015.48.3.242

Received 10 November 2014

Keywords: Cardiac hypertrophy, Histone deacetylases, Histone deacetylase inhibitors, Posttranslational modifications diomyocytes and rebirth of the fetal gene program without an apparent expansion of cardiomyocyte populations. Although the initial hypertrophy is physiological, sustained stimuli without alleviation of the underlying causal disease leads the heart to a pathological state (2). If the underlying disease continues without correction, the function of the hypertrophied heart declines, leading to heart failure. Despite intensive management, heart failure is still one of the most common causes of death (4).

To acquire proper functions, many proteins undergo modification after translation by enzymes, a process referred to as posttranslational modification (PTM). Indeed, diverse PTMs are involved in the regulation of the amount, function, and localization of many proteins. For example, phosphorylation is a critical modification for enzymatic activation (5), binding to DNA (6), forming complexes (7), and subcellular localization (8). Polyubiquitination, a continued link of ubiquitin to the lysine residue of a target protein or ubiquitin itself, affects protein stability, whereas monoubiqutination regulates other functions (9). These kinds of modifications are finely regulated by paired enzymes with opposite functions, such as kinases and phosphatases, methyltransferases and demethylases, acetyl transferases and deacetylases, and ubiquitinylases and deubiquitinylases.

Protein acetylation is also closely related to enzyme activity (10), DNA recruitment (11), and transcription (12). It typically occurs on lysine residues; however, $\mathrm{N}$-terminal acetylation has also been reported (13). This process replaces a hydrogen atom with acetyl moiety. Among acetylation-associated regulation, the biological implications of acetylation of histones $\mathrm{H} 3$ and $\mathrm{K} 4$ have been established; histone tail acetylation induces loosening of DNA from histones, leading to increased accessibility for the transcriptional machinery. Histone deacetylation, on the other hand, results in transcription silencing $(14,15)$. Histone acetylation is finely regulated by the balance between histone acetyl transferase and histone deacetylase (HDAC) activities.

\section{HDAC AND DIVERSE DISEASES}

Eighteen HDACs have been discovered in mammals and divided into four classes. HDAC $1 / 2 / 3 / 8$ are class I HDACs. HDAC4/5/6/7/9/10 are members of the class II HDACs. Class 
III HDACs (HDAC12-18) are classified as a sirtuin group and they require $\mathrm{NAD}^{+}$for intrinsic activity. HDAC11 is the only class IV HDAC. Class II HDACs are further subcategorized into two subclasses: HDAC4/5/7/9 for class Ila HDACs and HDAC6/ 10 for class Ilb. Molecular structures, tissue distributions, and subcellular localizations are summarized in Fig. 1.

Although class II HDACs have a conserved HDAC domain, it is somewhat controversy whether they actually have deacetylase activity in vivo $(16,17)$. Indeed, the role of class Ila HDACs is regarded as serving as a scaffold to form a larger complex for transcription repression. Additionally, class II HDACs interfere with binding of transcription activators, such as MEF2 (8, 18). Some reports, including ours, however, showed that the class Ila HDACs possess minute but significant intrinsic deacetylase activity in vivo $(19,20)$.

The subcellular localization of class Ila HDACs is controlled by exogenous signals $(8,21,22)$ : activated PKC/PKD or CaMKII phosphorylates serine residues that flank the nuclear localization signal domain. Shuttling molecules, such as 14-3-3 or CRM1, bind specifically to phosphorylated class lla HDACs, which results in the masking of the nuclear localization signal. This enables class Ila HDACs to exit to the cytoplasm. In contrast, information on the regulation of class Ilb HDACs is lim- ited; both HDAC6 and HDAC10 are primarily localized in the cytoplasm. HDAC6 specifically regulates acetylation of $\alpha$-tubulin and thereby microtubule assembly (23); however, the functional implications of HDAC6 remain to be clarified.

Although the molecular sizes of class I HDACs are relatively small, their enzyme activities are greater than those of class Ila HDACs. Class I HDACs are exclusively involved in protein deacetylation. Indeed, class I HDACs contribute most of total HDAC activity measured in vitro in whole cell lysates. HDAC1 and HDAC2 are major deacetylase enzymes of histones for transcriptional repression. Interestingly, HDAC1 redundantly regulates histone acetylation together with $\operatorname{HDAC2}(14,24)$.

The clinical implications of the roles of HDACs have been suggested based on the effects of HDAC inhibitors (HDACi) in various diseases. For example, vascular calcification (25) may be aggravated by HDAC inhibition. This suggests that loss of HDAC activity may play a key role in the development of the disease. On the other hand, a non-selective HDACi effectively arrests the cell cycle or induces apoptosis (26). For example, trichostatin A (TSA), a pan-HDACi, blocks smooth muscle cell proliferation, suggests that HDACi may have therapeutic potential for atherosclerosis and restenosis after stent insertion in coronary arteries (27). Numerous research groups have tested

\begin{tabular}{|c|c|c|c|c|}
\hline Class & \multicolumn{2}{|c|}{ Structure } & Subcelluar localization & Tissue distribution \\
\hline \multirow[t]{4}{*}{ I } & HDAC1 & HDAC & Nucleus & Ubiquitous \\
\hline & HDAC2 & HDAC & Nucleus & Ubiquitous \\
\hline & HDAC3 & HDAC & Nucleus/cytoplasm & Ubiquitous \\
\hline & HDAC8 & HDAC & Nucleus/cytoplasm & Ubiquitous \\
\hline \multirow[t]{4}{*}{ IIa } & HDAC4 & HDAC & Nucleus/cytoplasm & $\begin{array}{l}\text { Skeletal muscle, } \\
\text { Heart, Bone }\end{array}$ \\
\hline & HDAC5 & HDAC & Nucleus/cytoplasm & $\begin{array}{l}\text { Skeletal muscle, } \\
\text { Heart, Brain }\end{array}$ \\
\hline & HDAC7 & HDAC & Nucleus/cytoplasm & $\begin{array}{l}\text { Smooth muscle, } \\
\text { Placenta, Heart }\end{array}$ \\
\hline & HDAC9 & HDAC & Nucleus/cytoplasm & $\begin{array}{l}\text { Skeletal muscle, } \\
\text { Heart, Brain }\end{array}$ \\
\hline \multirow[t]{2}{*}{$\mathrm{IIb}$} & \multirow{2}{*}{$\begin{array}{l}\text { HDAC6 } \\
\text { HDAC10 }\end{array}$} & HDAC & Mainly cytoplasm & $\begin{array}{l}\text { Heart, Kidney, } \\
\text { Placenta, Liver }\end{array}$ \\
\hline & & HDAC & Mainly cytoplasm & $\begin{array}{l}\text { Liver, Kidney, } \\
\text { Spleen }\end{array}$ \\
\hline IV & HDAC11 & HDAC & Nucleus/cytoplasm & $\begin{array}{l}\text { Skeletal muscle. } \\
\text { Barin, Kidney }\end{array}$ \\
\hline
\end{tabular}

Fig. 1. Molecular structure, tissue distribution and subcellular localization of histone deacetylase. Color boxes. Red: MEF2C binding domain, Scarlet: PKD phosphorylation sites, Yellow: nuclear localization signal, Blue: nuclear export signal, Black: histone deacetylase domain. 
the anti-neoplastic properties of HDACi and the U.S. Food and Drug Administration has approved suberoylanilide hydroxamic acid and romidepsin for novel regimens against cutaneous $\mathrm{T}$ cell lymphoma. Sodium valproate, which has long been used as an anti-epileptic therapeutic, has undergone a phase II clinical trial for treating solid tumors (28).

In addition to anti-neoplastic activity, recent pioneering work has revealed that HDACi are also beneficial for diverse cardiac diseases: arrhythmia (29), myocardial infarction (30-32), hypertension (33), and cardiac hypertrophy (34-38). In the following section, we discuss 1) the role of HDACs and 2) the regulation mechanisms of HDACs in cardiac hypertrophy.

\section{HDACs AND CARDIAC HYPERTROPHY}

Class I HDACs are expressed ubiquitously, whereas class II HDACs are not. Among the class II HDACs, HDAC4/5/6/7/9 are expressed in the heart; however, the specific role of each subtypes is not yet known. The first understanding of class II HDACs' roles in the cardiac fields was in relation to HDAC9 (8). Based on the phenotype evaluation of whole-body deletion of HDAC9, it was suggested that HDAC9 may function as a negative regulator of cardiac hypertrophy; cardiac hypertrophy is dramatically exaggerated in HDAC9 knockout $(\mathrm{KO})$ mice.

MITR, an endogenous alternatively spliced form of HDAC9 that lacks the HDAC domain, successfully rescued the absence of HDAC9 in vivo. These observations suggest that the function of HDAC9 in vivo may be as a 'simple' transcriptional repressor in a deacetylase enzyme-independent manner. Although the HDAC5 KO phenotype is similar to that of $\operatorname{HDAC9}(21,22)$, in contrast to HDAC9, interestingly, HDAC5 has the function of an "enzyme" during the development of cardiac hypertrophy. Recently, we reported that HDAC5 deacetylates HDAC2, a pro-hypertrophic mediator, and thereby suppresses its activity (19).

Among the class I HDACs, both HDAC1 and HDAC2 redundantly regulate cardiac development in the embryo (24). Cardiac-specific KO of HDAC1 or HDAC2 did not affect cardiac morphogenesis, whereas double deletion of both HDAC1 and HDAC2 was lethal at postnatal 14 day due to severe dilated cardiomyopathy. HDAC2, however, is the major class I HDAC in the adult heart. At least in the development of cardiac hypertrophy, the enzyme activity of HDAC2 is solely increased in response to hypertrophic stimuli without alteration of its expression level (39). The subcellular localization of HDAC2 was not changed in the development of cardiac hypertrophy. Rather, PTM-dependent activation is a key regulatory mechanism $(19,35,39)$.

\section{THE ROLE OF HISTONE DEACETYLASE 2 IN CARDIAC DISEASE}

HDAC2's role in cardiac disease first emerged as a responsible factor for "homeodomain-only protein" (HOPX)-derived hyper- trophy (37). Kook et al. reported that cardiac-specific overexpression of atypical HOPX that lacked a DNA binding motif could induce cardiac hypertrophy. Cardiac hypertrophy induced by transgenic overexpression of HOPX was blocked completely by HDACi. Interestingly, HOPX requires HDAC2 to induce cardiac hypertrophy; transgenic mice that overexpress HOPX that do not interact with HDAC2 fail to induce cardiac hypertrophy. This study first reported that class I HDAC might also be involved in cardiac hypertrophy and that it counteracted the function of class Ila HDACs. To delineate the net effect of the simultaneous inhibition of both class I and class II HDACs, non-selective HDACi were used. We (36) and others $(38,40)$ observed that cardiac hypertrophy provoked by pressure overload or by hypertrophic agonists was inhibited successfully by pan-HDACi. In subsequent studies, our group further showed that class I-selective HDACi also successfully inhibited cardiac hypertrophy, as pan-HDACi did (36, 40), suggesting that class I HDACs are responsible for the anti-hypertrophic action of pan-HDACi. Next, we tried to find which subtype of class I HDAC was responsible for the development of cardiac hypertrophy and found that diverse hypertrophic stresses specifically activated HDAC2. Thus, we concluded that HDAC2 played a central role in cardiac hypertrophy. HDAC2 activation contributes to a common pathway that various hypertrophic stresses merge into and inhibition of HDAC2 may be a therapeutic target to reverse cardiac hypertrophy and concomitant heart failure.

A HDAC2 KO study reinforced the conclusion above. Trivedi et al. (41) reported that whole-body KO of HDAC2 resulted in partial lethality due to failure of myocardial maturation and a trabeculation defect in the perinatal period. Surviving HDAC2 KO mice recover growth and cardiac function at 2 months of age. In the adult, genetic disruption of HDAC2 resulted in resistance against hypertrophic stimuli induced by pressure overload, a $\beta$-adrenergic agonist, or crossing with HOPX transgenic mice. Gain-of-function of HDAC2, however, induced cardiac hypertrophy significantly, whereas transgenic overexpression of HDAC1 or HDAC3 failed to do so.

The heart provides oxygen and nutrients to the periphery by its rhythmic contraction, which is strictly controlled by an electrical conducting system. Some evidence has been reported that HDAC2 participates in the electrical physiology of the heart. Montgomery et al. performed gene ontology analyses from cardiac-specific disruption of both HDAC1 and HDAC2 and found dramatic upregulation of calcium channels: Cacna1h and Cacna2d2 (24). We also performed cDNA microarray studies to check the mRNAs altered by transgenic overexpression of HDAC2 in the heart and found that several channel subtypes for ion handling were dysregulated: Scn3b, Kcne1, Cacnb2, and Scn2a1 (unpublished data). Considering that dysfunction of Kcne1 is involved in hereditary familial arrhythmia, by causing long QT syndrome, and that loss of function of Scn3b leads to Burgada syndrome (42), alteration of HDAC2 activity may indeed participate in cardiac arrhythmia. 
HDACi effectively controls atrial arrhythmia. Ismat et al. (43) performed surface ECG in HOPX-null mice and observed P-wave widening, QRS-complex widening, and prolongation of the QT interval. Connexin40 was also decreased aberrantly in those mice. On the other hand, P-wave duration was prolonged and atrial refractory periods were shortened in HOPXtransgenic mice (29). TSA successfully reversed the conduction defect by normalization of connexin40. On the basis of a physical interaction between HOPX and HDAC2 in vivo, we assume that the anti-arrhythmogenic properties of TSA may be associated with inhibition of HDAC2 recruited by HOPX (37, 44).

\section{POSTTRANSLATIONAL MODIFICATION OF HISTONE DEACETYLASE 2 IN CARDIAC HYPERTROPHY}

Next, we sought to understand the regulatory mechanism of HDAC2 in cardiac hypertrophy. First, we found that diverse hypertrophic stresses specifically activated HDAC2 (39). By hypertrophic stimuli, heat shock protein (HSP) 70 was induced markedly to maintain HDAC2 activity. The requirement for HDAC2 activation in cardiac hypertrophy was further confirmed by use of an enzymatically inert mutant of HDAC2: HDAC2 H141A that loses HDAC activity due to destruction of the active pocket without altering interactions with other proteins. When HDAC2 H141A was overexpressed, no apparent cardiomyocyte hypertrophy was observed. Based on these observations, we concluded that increased HDAC2 activity was critical for hypertrophy.

Regarding the HDAC2 regulatory mechanism, however, no change in protein amount or mRNA expression was observed, raising the possibility of the involvement of PTM. Indeed, HDAC2 phosphorylation was increased significantly by hypertrophic stimuli. Using bioinformatics and by scrutinizing previous reports, four serine residues were picked as phosphorylation candidates: S394, S411, S424, and S424. By an in vitro kinase assay using synthetic peptides, we found that S394 and S411 phosphorylation were increased significantly in tissue lysates obtained from hypertrophied mouse heart. Interestingly, S422 and S424 were also basally phosphorylated in heart lysate from normal mice; however, we did not observe any further increase in phosphorylation of those residues with hypertrophy. Next, we observed that the intrinsic activity of HDAC2 was decreased significantly when S394, S422, or S424 was mutated, whereas the enzyme activity of HDAC2 was unchanged in the S411A mutant. According to these results, we concluded that basal activity of HDAC2 is maintained by S422 and S424 phosphorylation, whereas hypertrophy-associated phosphorylation and subsequent enzyme activation of HDAC2 were mediated by S394 phosphorylation. Indeed, in primary culture cardiomyocytes and in transgenic mice, overexpression of HDAC2 wild-type successfully induced hypertrophy, whereas HDAC2 S394A failed to do so. HDAC2 S394 phosphorylation and hypertrophic phenotypes were blocked by various casein kinase
(CK) 2 inhibitors and by CK2 $2 \alpha 1$ siRNA. Transgenic overexpression of CK2 $\alpha 1$ in the heart successfully induced hypertrophy, which was further exacerbated by crossing TgCK $2 \alpha 1$ with TgHDAC2 wild-type, but blunted by mating with TgHDAC2 S394A in vivo. Thus, CK2 $\alpha 1$-dependent phosphorylation of HDAC2 S394 is the key modification for both HDAC2 activation and cardiac hypertrophy (35).

We also found that HDAC2 itself could be regulated by acetylation dynamics. Various hypertrophic stresses acetylate HDAC2 at lysine 75 and acetylation activates HDAC2. HDAC2 $\mathrm{K} 75 \mathrm{R}$, an acetylation-resistant mutant that loses intrinsic activity, failed to induce hypertrophy. In contrast, the acetylationmimicking mutant of HDAC2, HDAC2 K75Q, showed an increase in enzyme activity and also possessed pro-hypertrophic properties.

We further examined whether an interplay between acetylation and phosphorylation existed in the regulation of HDAC2 activity; HDAC2 S394 phosphorylation was decreased significantly in HDAC2 K75R. On the other hand, HDAC2 K75 acetylation was unchanged in HDAC2 S394A. The intrinsic activity of HDAC2 K75R was not further downregulated by S394A. These results suggested that 1) HDAC2 K75 acetylation precedes and is mandatory for HDAC2 S394 phosphorylation and 2) the acetylation did not directly activate the intrinsic activity of HDAC2, but indirectly induced activation of HDAC2, by S394 phosphorylation.

We further tried to find the enzymes involved in HDAC2 acetylation. We observed that HDAC2 acetylation was finely balanced by pCAF and HDAC5, a class Ila HDAC. Both HDAC2 acetylation and phosphorylation were upregulated significantly in HDAC5 null mice. Phosphorylated HDAC5 underwent cytoplasmic redistribution after recognition by CRM1. Leptomycin B, a CRM1 inhibitor, interfered with the shuttling of HDAC5 to the cytoplasm and thereby showed anti-hypertrophic features by tethering HDAC5 in the nucleus. Adenoviral expression of HDAC2 K75Q, a constitutively acetylated form of HDAC2, overcame the anti-hypertrophic activity of leptomycin B. Agonist-induced cardiac hypertrophy in HDAC5 $\mathrm{KO}$ mice was alleviated by HDAC2 K75R delivery to the heart, delivered via the tail vein (19). To summarize, hypertrophic stresses activate both CK2 $\alpha 1$ and PKD. PKD phosphorylates class Ila HDACs and phospho-class Ila HDACs shuttle out to the cytoplasm. HDAC2 undergoes acetylation by pCAF and then phosphorylation by activated-CK2 $\alpha 1$. Alternatively, hypertrophic stresses also induce the expression of HSP70. HSP70 recognizes and binds to phospho-HDAC2, which results in protection of HDAC2 phosphorylation against phosphatases (Fig. 2).

\section{DEACETYLATION TARGET OF ACTIVATED HDAC2 IN THE DEVELOPMENT OF CARDIAC HYPERTROPHY}

So, which signal cascade relays activation of HDAC2 to hypertrophic phenotypes in the cardiomyocytes? Trivedi et al. (41) 
performed DNA microarray assays to assess dysregulated genes in HDAC2 $\mathrm{KO}$ mice and they found inositol polyphosphate-5-phosphatase F (INPP5F), a negative regulator of the PI3K system. Genetic disruption of HDAC2 in the heart resulted in an increase in expression of the gene encoding INPP5F, which then resulted in inactivation of AKT and then reactivation of GSK3 3 . Next, Zhu et al. (45) generated wholebody deletions of INPP5F in the mouse and found that isoproterenol-induced cardiac hypertrophy was exaggerated significantly. Thus, INPP5F works as a negative regulator of cardiac hypertrophy by serving as a target of activated HDAC2. However, genetic ablation of INPP5F in vivo did not spontaneously cause hypertrophy, suggesting that INPP5F is not a unique target of activated HDAC2. Indeed, we found Krüppel-like factor 4 (KLF4) was an alternate target of activated HDAC2. To find HDAC2- resposive elements and the transcription factor responsible, we performed promoter mapping analyses with the natriuretic peptide precursor $A$ (encoding atrial natriuretic factor, ANF) promoter. The anti-hypertrophic action of $\mathrm{HDACi}$ was abolished when the region from -130 to -105 was deleted. Among the putative binding factors on this HDACi-responsive element, the expression level of KLF4 was altered both by $\mathrm{HDACi}$ and agonist stimuli. The expression of KLF4 and thereby binding on the Nppa-promoter were decreased significantly in the activation of HDAC2 induced by hypertrophic agonists or by HDAC2 transfection. Forced expression of KLF4 in the cardiomyocyte blunted hypertrophy. Thus, we concluded that HDAC2 activation regulated the transcription level of KLF4, a negative regulator of cardiac hypertrophy (46) (Fig. 2). Other groups have also reported the anti-hypertrophic function of KLF4 in vivo. Liao et al. (47) observed that cardiac hypertrophy was exacerbated in mice with cardiomyocyte-specific deletion of KLF4.

It has been reported that numerous proteins undergo acetylation and their unique functions are finely regulated by acetylation dynamics $(10-12,48)$. It has not been fully understood which enzymes are associated with the deacetylation process; however, several HDACs target non-histone proteins (23, 49-53). Indeed, HDAC2 also has non-histone substrates, such as the glucocorticoid receptor, BCL6, STAT3, and YY1. In addition to transcription-dependent regulation targets, such as INPP5F $(41,45)$ and KLF4 $(46)$, as above, it is possible that activated HDAC2 relays signal to non-histone target(s) in the development of cardiac hypertrophy. Considering that the cardiac-specific transcription factor GATA4 is involved in HOPX-HDAC2 signals in cardiac morphogenesis (44), acetylated GATA4 might be one of the novel targets for HDAC2 in cardiac hypertrophy.

\section{DISCUSSION}

We have clarified the role of HDAC2 and its regulatory mechanism in cardiac hypertrophy (Fig. 2). When the myocardium is stimulated by various exogenous stresses, numerous signal cascades are activated. For example, PKC/PKD is activated and phosphorylates class Ila HDACs. HSP70 is induced dramati-

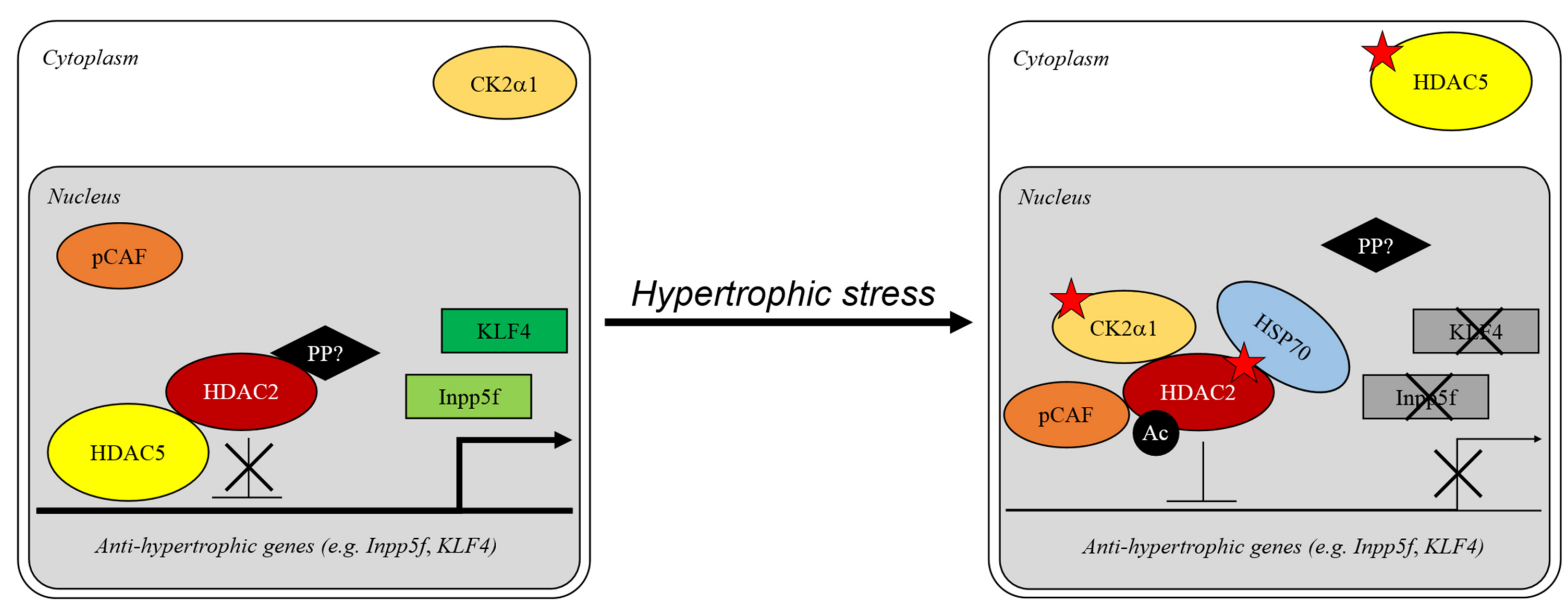

Fig. 2. Working hypothesis. In the basal condition, HDAC2 interacts with HDAC5 and protein phosphatase. Both HDAC5 and protein phosphatase keep HDAC2 inactivated. Loss of intrinsic activity of HDAC2 leads to de-repression of anti-hypertrophic gene activation, such as INPP5F or KLF4. The cardiac fetal gene program remains arrested by the inhibition of INPP5F or KLF4 (left). When the myocardium is stimulated by hypertrophic stresses, several signaling cascades operate. Class Ila HDACs, including HDAC5, are phosphorylated by PKD and undergo cytoplasmic redistribution after binding with 14-3-3. At the same time, CK2 $\alpha 1$ is also activated, by phosphorylation, and is shuttled into the nucleus. Inducible heat shock protein, HSP70, is induced in the nucleus. pCAF binds to and deacetylates HDAC2, which allows CK2 $\alpha 1$ to phosphorylate HDAC2 at serine 394. HSP70 specifically binds to phosphorylated HDAC2. Functionally activated HDAC2 suppresses the expression of negative regulators of hypertrophy, which induces reactivation of the fetal gene program. Red stars indicate phosphorylation. Abbreviations. Ac: acetylation, CK2 $\alpha 1$ : casein kinase 2 $\alpha 1$, HDAC: histone deacetylase, HSP70: heat shock protein 70, INPP5F: inositol polyphosphate-5-phosphatase F, KLF4: Krüppel-like factor 4, pCAF: p300/CBP-associated factor, PP: protein phosphatase. 
cally in the cytoplasm and pCAF is also activated in a PTM-dependent manner in the nucleus. Phosphorylated class Ila HDACs are captured by shuttling molecules and redistributed into the cytoplasm, whereas HSP70 shuttles into the nucleus. pCAF acetylates HDAC2 K75, which then allows CK2 $\alpha 1$ to phosphorylate HDAC2 S394. Activated HDAC2 is then recruited on promoters that drive the expression of anti-hypertrophic mediators, such as INPP5F or KLF4, which, in turn, downregulates the expression of those mediators by deacetylation of histones associated with their promoters. Downregulation of anti-hypertrophic regulators results in reactivation of an arrested fetal gene program, thereby resulting in cardiac hypertrophy.

Cardiac hypertrophy is a double-edged sword. Hypertrophy, a form of cardiac remodeling, however, is also an essential adaptation for increased hemodynamic demands, at least at the beginning of a cardiac compensation period. The transition mechanism from physiological to pathological hypertrophy remains unclear. We observed the enzyme activity of HDAC2 peaked prior to the apparent hypertrophy and returned to basal levels in the heart failure phase (39). Considering that increased HDAC2 activity is a key step in cardiac hypertrophy, the abrupt loss of HDAC2 activity might be a cue signal to transit to heart failure. A study using HDAC2 activators would be necessary to assess this.

We found a novel crosstalk between class I and class II HDACs in vivo. Fischle et al. (16) also reported the formation of a functional complex including class I and class II HDACs. The class II HDAC recruits and uses the class I HDAC to deacetylate promoters. In that model, however, the class II HDAC does not serve as an enzyme but as a scaffold. Moreover, it is also believed that diverse signaling cascades converge into the two classes HDACs and both classes of HDACs regulate cardiac hypertrophy independently. We observed, however, that HDAC5 does have intrinsic activity and the enzyme activity of HDAC5 is indispensable for the deacetylation of HDAC2 and that the HDACs-mediated signal cascade, considered as two independent axes, can merge into a single stream.

\section{FUTURE ASPECTS}

Other types of PTM of HDAC2 beyond phosphorylation and acetylation have also been reported in various disease models (54-57). Colussi et al. (55) reported that HDAC2 was significantly upregulated in Duchenne muscular dystrophy and blockade by HDACi or cysteine-S-nitrosylation was beneficial to improve dystrophy symptoms. Nott et al. (54) observed in neuronal development that S-nitrosylation of HDAC2 induced its dissociation from DNA, which then resulted in histone acetylation and then transcription activation. Thus, it is plausible that the S-nitrosylation modification of HDAC2 is a potent modification to suppress its activity. Recently, HDAC2 sumoylation has also been reported (57), which causes enzymatic activation.
We assume that dephosphorylation may participate in the inactivation of HDAC2 $(19,35,39)$. Though a hypertrophy- associated phosphatase of HDAC2 has not been identified, in other disease conditions, some phosphatases have been suggested as HDAC2 phosphatases (58-60). The total phosphorylation level of HDAC2 is increased significantly by simultaneous treatment with okadaic acid, a protein phosphatase $2 \mathrm{~A}$ (PP2A) inhibitor. In our models, we also observed that the catalytic subunit of PP2A interacted physically with HDAC2. PP2A reduced both phosphorylation and the intrinsic activity of HDAC2 significantly. Overexpression of PP2A blocked cardiomyocyte hypertrophy induced by HDAC2 (Min et al., unpublished observations)

We $(19,27,34-37,39,46)$ and others $(38,40,41,45)$ have demonstrated the functional relevance of HDAC2 and cardiac remodeling and HDAC2 may be a promising target for cardiac remodeling. One may argue, however, that unwanted effect would be expected given the ubiquitous distribution of HDAC2, which may restrict its therapeutic use. However, as shown in the previous report (41), HDAC2 is exclusively expressed in the developing heart. Moreover, considering that HDACs work in a combinatorial fashion with diverse transcription factors that determine the downstream target and that these combinations would vary greatly depending on cellular context and/or disease processes, tissue specificity would be achieved by simultaneous modulation of these factors as well as their cellular contexts. Thus, understanding these complex pathways and the functions of the associated transcription factors would be of great importance for the specificity of HDAC modulation. In addition, it is important to understand the PTM of HDAC and their regulators. On the basis of knowledge that intrinsic of HDACs can be regulated by dynamics of diverse PTMs, modifiers of HDACs could also provide notable therapeutic targets by minimizing the adverse effects of HDACi. Indeed, we have already shown that anti-hypertrophic properties of CK2 inhibitors, which interfered with HDAC2 phosphorylation, was as effective as HDACi in vivo (35). Like CK2 inhibitors, certain chemicals targeted downstream of HDAC2 could be important for the treatment of cardiac remodeling.

\section{ACKNOWLEDGEMENTS}

This study was supported by a National Research Foundation of Korea grant, funded by the Korean government (MEST, \#2012-0005602), and by a grant from the Korean Health Technology R\&D Project, Ministry of Health \& Welfare, Republic of Korea (A121561).

\section{REFERENCES}

1. Eom GH and Kook H (2014) Posttranslational modifications of histone deacetylases: implications for cardiovascular diseases. Pharmacol Ther 143, 168-180

2. Frey N and Olson EN (2003) Cardiac hypertrophy: the 
good, the bad, and the ugly. Annu Rev Physiol 65, 45-79

3. Hill JA and Olson EN (2008) Cardiac plasticity. N Engl J Med 358, 1370-1380

4. Hunter DJ and Reddy KS (2013) Noncommunicable diseases. N Engl J Med 369, 1336-1343

5. Dimmeler S, Fleming I, Fisslthaler B, Hermann C, Busse R and Zeiher AM (1999) Activation of nitric oxide synthase in endothelial cells by Akt-dependent phosphorylation. Nature 399, 601-605

6. Beg AA, Finco TS, Nantermet PV and Baldwin AS Jr (1993) Tumor necrosis factor and interleukin-1 lead to phosphorylation and loss of I kappa B alpha: a mechanism for NF-kappa B activation. Mol Cell Biol 13, 3301-3310

7. Maudsley S, Pierce KL, Zamah AM et al (2000) The be$\mathrm{ta}(2)$-adrenergic receptor mediates extracellular signalregulated kinase activation via assembly of a multi-receptor complex with the epidermal growth factor receptor. J Biol Chem 275, 9572-9580

8. Zhang CL, McKinsey TA, Chang S, Antos CL, Hill JA and Olson EN (2002) Class II histone deacetylases act as signal-responsive repressors of cardiac hypertrophy. Cell 110, 479-488

9. Park CW and Ryu KY (2014) Cellular ubiquitin pool dynamics and homeostasis. BMB Rep 47, 475-482

10. Santos-Rosa H, Valls E, Kouzarides T and Martinez-Balbas M (2003) Mechanisms of P/CAF auto-acetylation. Nucleic Acids Res 31, 4285-4292

11. Gu W and Roeder RG (1997) Activation of p53 sequence-specific DNA binding by acetylation of the p53 C-terminal domain. Cell 90, 595-606

12. Evans PM, Zhang W, Chen X, Yang J, Bhakat KK and Liu C (2007) Kruppel-like factor 4 is acetylated by p300 and regulates gene transcription via modulation of histone acetylation. J Biol Chem 282, 33994-34002

13. Driessen HP, de Jong WW, Tesser Gl and Bloemendal $\mathrm{H}$ (1985) The mechanism of N-terminal acetylation of proteins. CRC Crit Rev Biochem 18, 281-325

14. Laherty CD, Yang WM, Sun JM, Davie JR, Seto E and Eisenman RN (1997) Histone deacetylases associated with the mSin3 corepressor mediate mad transcriptional repression. Cell 89, 349-356

15. Nagy L, Kao HY, Chakravarti D et al (1997) Nuclear receptor repression mediated by a complex containing SMRT, mSin3A, and histone deacetylase. Cell 89, 373-380

16. Fischle W, Dequiedt $F$, Hendzel MJ et al (2002) Enzymatic activity associated with class II HDACs is dependent on a multiprotein complex containing HDAC3 and SMRT/N-CoR. Mol Cell 9, 45-57

17. Jones P, Altamura S, De Francesco R et al (2008) Probing the elusive catalytic activity of vertebrate class Ila histone deacetylases. Bioorg Med Chem Lett 18, 1814-1819

18. McKinsey TA, Zhang CL and Olson EN (2000) Activation of the myocyte enhancer factor- 2 transcription factor by calcium/calmodulin-dependent protein kinase-stimulated binding of 14-3-3 to histone deacetylase 5. Proc Natl Acad Sci U S A 97, 14400-14405

19. Eom GH, Nam YS, Oh JG et al (2014) Regulation of acetylation of histone deacetylase 2 by p300/CBP-associated factor/histone deacetylase 5 in the development of cardiac hypertrophy. Circ Res 114, 1133-1143

20. Nebbioso A, Manzo F, Miceli M et al (2009) Selective class II HDAC inhibitors impair myogenesis by modulating the stability and activity of HDAC-MEF2 complexes. EMBO Rep 10, 776-782

21. Chang S, McKinsey TA, Zhang CL, Richardson JA, Hill JA and Olson EN (2004) Histone deacetylases 5 and 9 govern responsiveness of the heart to a subset of stress signals and play redundant roles in heart development. Mol Cell Biol 24, 8467-8476

22. Vega RB, Harrison BC, Meadows E et al (2004) Protein kinases $C$ and $D$ mediate agonist-dependent cardiac hypertrophy through nuclear export of histone deacetylase 5. Mol Cell Biol 24, 8374-8385

23. Hubbert C, Guardiola A, Shao R et al (2002) HDAC6 is a microtubule-associated deacetylase. Nature 417, 455-458

24. Montgomery RL, Davis CA, Potthoff MJ et al (2007) Histone deacetylases 1 and 2 redundantly regulate cardiac morphogenesis, growth, and contractility. Genes Dev 21, 1790-1802

25. Azechi T, Kanehira D, Kobayashi T et al (2013) Trichostatin $\mathrm{A}$, an HDAC class $\mathrm{I} / \mathrm{II}$ inhibitor, promotes pi-induced vascular calcification via up-regulation of the expression of alkaline phosphatase. J Atheroscler Thromb 20, 538-547

26. Johnstone RW (2002) Histone-deacetylase inhibitors: novel drugs for the treatment of cancer. Nat Rev Drug Discov $1,287-299$

27. Kee HJ, Kwon JS, Shin S, Ahn Y, Jeong MH and Kook H (2011) Trichostatin A prevents neointimal hyperplasia via activation of Kruppel like factor 4. Vascul Pharmacol 55, $127-134$

28. Cincarova L, Zdrahal Z and Fajkus J (2013) New perspectives of valproic acid in clinical practice. Expert Opin Investig Drugs 22, 1535-1547

29. Liu F, Levin MD, Petrenko NB et al (2008) Histone-deacetylase inhibition reverses atrial arrhythmia inducibility and fibrosis in cardiac hypertrophy independent of angiotensin. J Mol Cell Cardiol 45, 715-723

30. Zhao TC, Cheng G, Zhang LX, Tseng YT and Padbury JF (2007) Inhibition of histone deacetylases triggers pharmacologic preconditioning effects against myocardial ischemic injury. Cardiovasc Res 76, 473-481

31. Lee TM, Lin MS and Chang NC (2007) Inhibition of histone deacetylase on ventricular remodeling in infarcted rats. Am J Physiol Heart Circ Physiol 293, H968-977

32. Granger A, Abdullah I, Huebner F et al (2008) Histone deacetylase inhibition reduces myocardial ischemia-reperfusion injury in mice. FASEB J 22, 3549-3560

33. Lee HA, Lee DY, Cho HM, Kim SY, Iwasaki Y and Kim IK (2013) Histone deacetylase inhibition attenuates transcriptional activity of mineralocorticoid receptor through its acetylation and prevents development of hypertension. Circ Res 112, 1004-1012

34. Cho YK, Eom GH, Kee HJ et al (2010) Sodium valproate, a histone deacetylase inhibitor, but not captopril, prevents right ventricular hypertrophy in rats. Circ J 74, 760-770

35. Eom GH, Cho YK, Ko JH et al (2011) Casein kinase-2alpha1 induces hypertrophic response by phosphorylation of histone deacetylase 2 S394 and its activation in the heart. Circulation 123, 2392-2403 
36. Kee HJ, Sohn IS, Nam KI et al (2006) Inhibition of histone deacetylation blocks cardiac hypertrophy induced by angiotensin II infusion and aortic banding. Circulation 113, 51-59

37. Kook H, Lepore JJ, Gitler AD et al (2003) Cardiac hypertrophy and histone deacetylase-dependent transcriptional repression mediated by the atypical homeodomain protein Hop. J Clin Invest 112, 863-871

38. Kong Y, Tannous P, Lu G et al (2006) Suppression of class I and II histone deacetylases blunts pressure-overload cardiac hypertrophy. Circulation 113, 2579-2588

39. Kee $\mathrm{HJ}$, Eom $\mathrm{GH}$, Joung $\mathrm{H}$ et al (2008) Activation of histone deacetylase 2 by inducible heat shock protein 70 in cardiac hypertrophy. Circ Res 103, 1259-1269

40. Gallo P, Latronico MV, Gallo P et al (2008) Inhibition of class I histone deacetylase with an apicidin derivative prevents cardiac hypertrophy and failure. Cardiovasc Res 80 , 416-424

41. Trivedi CM, Luo Y, Yin Z et al (2007) Hdac2 regulates the cardiac hypertrophic response by modulating Gsk3 beta activity. Nat Med 13, 324-331

42. Monteforte N, Napolitano C and Priori SG (2012) Genetics and arrhythmias: diagnostic and prognostic applications. Rev Esp Cardiol (Engl Ed) 65, 278-286

43. Ismat FA, Zhang M, Kook $\mathrm{H}$ et al (2005) Homeobox protein Hop functions in the adult cardiac conduction system. Circ Res 96, 898-903

44. Trivedi CM, Zhu W, Wang Q et al (2010) Hopx and Hdac2 interact to modulate Gata4 acetylation and embryonic cardiac myocyte proliferation. Dev Cell 19, 450-459

45. Zhu W, Trivedi CM, Zhou D, Yuan L, Lu MM and Epstein JA (2009) Inpp5f is a polyphosphoinositide phosphatase that regulates cardiac hypertrophic responsiveness. Circ Res 105, 1240-1247

46. Kee HJ and Kook H (2009) Kruppel-like factor 4 mediates histone deacetylase inhibitor-induced prevention of cardiac hypertrophy. J Mol Cell Cardiol 47, 770-780

47. Liao X, Haldar SM, Lu Y et al (2010) Kruppel-like factor 4 regulates pressure-induced cardiac hypertrophy. J Mol Cell Cardiol 49, 334-338

48. Levy L, Wei Y, Labalette C et al (2004) Acetylation of beta-catenin by $\mathrm{p} 300$ regulates beta-catenin-Tcf4 interaction. Mol Cell Biol 24, 3404-3414

49. Chen LF, Mu Y and Greene WC (2002) Acetylation of
RelA at discrete sites regulates distinct nuclear functions of NF-kappaB. EMBO J 21, 6539-6548

50. Ito A, Kawaguchi Y, Lai CH et al (2002) MDM2-HDAC1mediated deacetylation of p53 is required for its degradation. EMBO J 21, 6236-6245

51. Ito K, Yamamura S, Essilfie-Quaye S et al (2006) Histone deacetylase 2-mediated deacetylation of the glucocorticoid receptor enables NF-kappaB suppression. J Exp Med 203, 7-13

52. Watamoto K, Towatari M, Ozawa Y et al (2003) Altered interaction of HDAC5 with GATA-1 during MEL cell differentiation. Oncogene 22, 9176-9184

53. Choudhary C, Kumar C, Gnad F et al (2009) Lysine acetylation targets protein complexes and co-regulates major cellular functions. Science 325, 834-840

54. Nott $A$, Watson PM, Robinson JD, Crepaldi $L$ and Riccio A (2008) S-Nitrosylation of histone deacetylase 2 induces chromatin remodelling in neurons. Nature 455, 411-415

55. Colussi C, Mozzetta C, Gurtner A et al (2008) HDAC2 blockade by nitric oxide and histone deacetylase inhibitors reveals a common target in Duchenne muscular dystrophy treatment. Proc Natl Acad Sci U S A 105, 19183-19187

56. Malhotra D, Thimmulappa RK, Mercado N et al (2011) Denitrosylation of HDAC2 by targeting Nrf2 restores glucocorticosteroid sensitivity in macrophages from COPD patients. J Clin Invest 121, 4289-4302

57. Brandl A, Wagner T, Uhlig KM et al (2012) Dynamically regulated sumoylation of HDAC2 controls p53 deacetylation and restricts apoptosis following genotoxic stress. J Mol Cell Biol 4, 284-293

58. Galasinski SC, Resing KA, Goodrich JA and Ahn NG (2002) Phosphatase inhibition leads to histone deacetylases 1 and 2 phosphorylation and disruption of corepressor interactions. J Biol Chem 277, 19618-19626

59. Adenuga D and Rahman I (2010) Protein kinase CK2-mediated phosphorylation of HDAC2 regulates co-repressor formation, deacetylase activity and acetylation of HDAC2 by cigarette smoke and aldehydes. Arch Biochem Biophys 498, 62-73

60. Adenuga D, Yao H, March TH, Seagrave J and Rahman I (2009) Histone deacetylase 2 is phosphorylated, ubiquitinated, and degraded by cigarette smoke. Am J Respir Cell Mol Biol 40, 464-473 